\title{
Synchrotron Methods of the Conduction Band Electronic Structure Analysis
}

\author{
A. KISIEL \\ Marian Smoluchowski Institute of Physics, Jagiellonian University \\ Reymonta 4, 30-059 Kraków, Poland
}

The experimental and theoretical studies of the density of states for unoccupied conduction band are a valid source of knowledge on the electronic structure of condensed matter. Among known analytical methods of density of states for unoccupied states a significant place is occupied by the methods with the use of intensive synchrotron radiation beam. Adequately chosen monochromatic beam excites electrons from the occupied core states or the valence band to unoccupied states in the conduction band. The most universal experimental method for the studies of the unoccupied conduction band is the X-ray absorption near edge structure analysis for X-ray edges of the constituent atoms of the material. A satisfying description of the unoccupied conduction band density of states can be also reached by the analysis of reflectivity coefficient in the vacuum ultraviolet (above $10 \mathrm{eV}$ ). The photoelectron energy distribution curves elaborated by the constant initial state and constant final state procedures deliver valuable information on the high excited states of conduction band. In the paper the menaces of the correct interpretation of the experimental results will be discussed. The efficiency of the unoccupied conduction band density of states studies with application of $\mathrm{X}$-ray absorption near edge structure and analysis of reflectivity coefficient of light will be presented on the basis of several binary and ternary semiconductor compounds of II-VI group with transition metals as well as of some other compounds. To avoid a misinterpretation of the experimental results, the data were compared and discussed with an adequate theoretical band structure calculation.

PACS numbers: 71.20.-b, 71.23.+i, 71.55.Gs, 78.20.Ci, 78.70.Dm

\section{Introduction}

A knowledge of the unoccupied electronic state properties of the conductivity band has a large significance for the understanding of many valid solid state features. For the solid state in the thermal equilibrium, when $T \rightarrow 0 \mathrm{~K}$, the unoccupied electronic states are separated from the occupied core and valence electronic states by the Fermi energy level defined by the Fermi-Dirac 
statistic $[1-3]$. For $T \neq 0 \mathrm{~K}$ in the Fermi-Dirac statistic the border between occupied and unoccupied states is described by the chemical potential. The experimental studies of the unoccupied state properties can be carried out with the use of electrical and thermal conductivity analysis as well as electrical and thermal conductivity studies in magnetic field. The application of methods described in the monographs and textbooks, for instance in Refs. [1-3] provide the valuable information on the conduction of unoccupied bands close to the centre of the Brillouin zone (BZ) or as in case of the cyclotron resonance analysis, provide a selected piece of information on the curvature of the maxima or minima in the critical points inside of the first BZ. Besides, the mentioned above experimental methods which are the sources of information on the unoccupied states structure and their density of states, can be applied with full success for the analysis of absorption and reflectivity of light [4-6], X-ray absorption near edge structure (XANES) [7-10], as well as for application of ultraviolet and X-ray photoemission spectroscopy (UPS and XPS) [11], inverted photoemission spectroscopy (IPS) [10], bremsstrahlung isochromat spectroscopy (BIS) [10] and appearance potential spectroscopy (APS) [10]. To the advanced studies of the fundamental reflectivity spectroscopy in the wide energy range from about 2 to $30 \mathrm{eV}$ and XANES analysis it is necessary to use strong intensity light sources with the continuous wave length spectrum. This condition is satisfied by the synchrotron radiation emitted in the electron or positron synchrotrons [11-13]. In this paper we will focus only on the unoccupied state analysis with an application of the fundamental reflectivity of light and XANES analysis. Both mentioned above experimental techniques deliver valuable and wide information on the unoccupied states of the solid state. In the last twenty years the hundreds of papers on the XANES applications have been published, thus a selection of the most characteristic applications is not so easy. Therefore, only several characteristic XANES spectrum properties and the application of XANES analysis will be presented. The fundamental reflectivity of light and XANES analysis on some II-VI group binary and multinary semiconducting compounds will be illustrated.

\section{Analysis of the fundamental reflectivity of light}

It is well known that absorption and reflectivity of light can deliver basic information on the electronic structure of solid state. This information for semiconducting materials can be obtained from the analysis of the absorption and reflectivity coefficients in a very wide energy range from the energy lower than the forbidden energy gap $E_{\mathrm{g}}$ up to about $30 \mathrm{eV}$. The reflectivity of light in this wide energy range is commonly named a fundamental reflectivity. The fundamental reflectivity describes the electronic transitions in which dominate two types of transitions:

1. the interband electronic transition from the occupied by electrons valence band to the unoccupied electronic state of conduction band, and 
2. the electron transition from the outside atomic core level to the unoccupied states of the conduction band.

\subsection{Interband electronic transitions}

The interband electronic transitions from the occupied by electrons valence band to the unoccupied conductivity band can be described by the electron transition probability [4-6]. In one-electron approximation* the electron excitation probability of the valence electron described by the energy $E_{\mathrm{v}}(\boldsymbol{k})$ and momentum vector $\boldsymbol{k}$ excited to the respective branch of the conduction band, characterised by energy $E_{\mathrm{c}}\left(\boldsymbol{k}^{\prime}\right)$ and momentum $\boldsymbol{k}^{\prime}$ is expressed by the formula $[4,6]$ :

$$
P\left(\omega, t, \boldsymbol{k}, \boldsymbol{k}^{\prime}\right)=\frac{e^{2}}{n^{2} h^{2}}\left(\left|\int \mathrm{d} t \int_{V} \mathrm{~d} \boldsymbol{r} \psi_{\mathrm{c}}\left(\boldsymbol{k}^{\prime}, \boldsymbol{r}\right) \boldsymbol{A} \cdot \boldsymbol{p} \psi_{\mathrm{v}}(\boldsymbol{k}, \boldsymbol{r})\right|\right) .
$$

In this formula the scalar product $\boldsymbol{A} \cdot \boldsymbol{p}$ is a linear approximation of the electromagnetic wave interaction with electron described by a momentum operator $\boldsymbol{p}$. The vector potential electromagnetic wave is expressed by formula $\boldsymbol{A}=A_{0} \boldsymbol{e} \mathrm{e}^{\mathrm{i}(\boldsymbol{\eta} \cdot \boldsymbol{r}-\omega t)}$, where $\boldsymbol{\eta}$ and $\boldsymbol{e}$ are wave vector and polarisation vector of the electromagnetic wave, respectively. The functions $\psi_{\mathrm{c}}\left(\boldsymbol{k}^{\prime}, \boldsymbol{r}\right)$ and $\psi_{\mathrm{v}}(\boldsymbol{k}, \boldsymbol{r})$ are the Bloch functions of collective electronic states for the valence and conductivity bands in solid state, respectively. In order to obtain the number of the transitions $W(\omega)$ per time and per volume unit induced by light of frequency $\omega$ one must sum over all possible states in the unit volume i.e. one must sum over $\boldsymbol{k}$, spin variable $s$ and over all indices of the valence v (occupied) and conductivity c (unoccupied) bands. After some conversions leading to expression of the formulae in terms of the $\boldsymbol{k}$ vector space and a change of the integration limits in formula (1) the number of transitions $W(\omega)$, limited only to the direct electron transitions (for $\boldsymbol{k}=\boldsymbol{k}^{\prime}$ ), can be expressed as

$$
W(\omega)=\frac{2 \pi}{h}\left(\frac{e A_{0}}{m c}\right)^{2} \sum_{\mathrm{v}, \mathrm{c}} \int_{\mathrm{BZ}} \frac{2 \mathrm{~d} k}{(2 \pi)^{3}}\left|\boldsymbol{e} \cdot \boldsymbol{M}_{\mathrm{cv}}(\boldsymbol{k})\right|^{2} \delta\left[E_{\mathrm{c}}(\boldsymbol{k})-E_{\mathrm{v}}(\boldsymbol{k})-\omega\right],
$$

where the integral extends over the first BZ and $\boldsymbol{e} \cdot \boldsymbol{M}_{\mathrm{cv}}(\boldsymbol{k})=\left\langle\Psi_{\mathrm{c}}\left|\mathrm{e}^{\mathrm{i} \boldsymbol{k} \boldsymbol{r}} \boldsymbol{e} \cdot \boldsymbol{p}\right| \Psi_{\mathrm{v}}\right\rangle$ are the matrix elements of the electron transition from any original valence band state described by the wave function $\Psi_{\mathrm{v}}$ to any final state in the conduction band, described by the wave function $\psi_{\mathrm{c}} . E_{\mathrm{v}}(\boldsymbol{k})$ and $E_{\mathrm{c}}(\boldsymbol{k})$ are the eigenvalues of the valence and conductive band branches, respectively. The factor 2 in formula (2) arises from integration of spin variables.

\footnotetext{
${ }^{*}$ In the more careful analysis an excitation of the electron from the occupied state to the unoccupied state it should be taken into consideration the interaction between a hole created in the occupied valence band and the excited electron. The calculated density of state distribution of the unoccupied states in the conduction band without and with the electron-hole interaction are different [1]. Unfortunately, a precise inclusion of the electron-hole interaction to the theoretical band structure calculations is complicated, thus in this paper we limit a comparison of the presented experimental data with theoretical calculations made only in one-electron approximation which, in general, sufficiently describes the occupied and unoccupied bands and their density of states.
} 
A number of transition $W(\omega)$ described by formula (2) is closely defined with the absorption coefficient of electromagnetic wave in the solid state by formula

$$
\mu(\omega)=\frac{\hbar \omega W(\omega)}{\rho(c / n)},
$$

where: the numerator $\hbar \omega W(\omega)$ is the energy absorbed per unit volume and time, and the denominator $\rho(c / n)$ is the energy flux, which is the product of the energy density and the velocity of propagation in the medium. The absorption coefficient defines the energy loss in the matter and it is bounded with the imaginary part $\epsilon_{2}$ of the complex dielectric function $\epsilon$ by formula

$$
\mu(\omega)=\frac{\omega}{n c} \varepsilon_{2} .
$$

Taking into account formulae (2), (3) and (4) $\epsilon_{2}$ can be expressed by

$$
\epsilon_{2}(\omega)=\frac{4 \pi^{2} e^{2}}{m^{2} \omega^{2}} \sum_{\mathrm{v}, \mathrm{c}} \int_{\mathrm{BZ}} \frac{2 \mathrm{~d} k}{(2 \pi)^{3}}\left|\boldsymbol{e} \cdot \boldsymbol{M}_{\mathrm{cv}}(\boldsymbol{k})\right|^{\delta}\left[E_{\mathrm{c}}(\boldsymbol{k})-E_{\mathrm{v}}(\boldsymbol{k})-\omega\right] .
$$

This formula is very useful for the comparison of the experimental and theoretical calculations. The intensity of absorbed light $I_{\mathrm{A}}$ is closely bounded with the intensity of reflected light $I_{\mathrm{R}}$ by the simple relation $I_{0}=I_{\mathrm{R}}+I_{\mathrm{T}}+I_{\mathrm{A}}$, where additionally $I_{0}$ and $I_{\mathrm{T}}$ are incident and transmitted light, respectively. Using the commonly known formulae defining absorption and reflection coefficients and applying the Kramers-Kronig relations one can calculate these optical constants in relation of the electron transition probability.

The matrix elements between couple of valence and conduction bands can be shown as a smooth function of $\boldsymbol{k}$ vector, thus it can be accepted as a constant. Applying to Eq. (3) the properties of $\delta$ function one can obtain in the absorption coefficient the contribution from a pair of bands which is proportional to $1 / \omega^{2}$ and to the quantity called the function of the join density of states

$$
\mu(\omega)=M J_{\mathrm{vc}} / \omega^{2},
$$

where

$$
J_{\mathrm{vc}}=\frac{2}{(2 \pi)^{2}} \int_{E_{\mathrm{c}}(k)-E_{\mathrm{v}}(k)=E} \frac{\mathrm{d} S}{\operatorname{grad}\left(E_{\mathrm{c}}-E_{\mathrm{v}}\right)} .
$$

In this formula $\mathrm{d} S$ is the element of the surface in the $\boldsymbol{k}$ vector space defined by equation $E_{\mathrm{c}}(\boldsymbol{k})-E_{\mathrm{v}}(\boldsymbol{k})=E$, it means that an integration extends over surfaces of constant energy. From expression (7) it is easy to see that the function of join density of states (JDOS) $J_{\mathrm{vc}}$ has singularities, named also the critical points, when

$$
\operatorname{grad}\left(E_{\mathrm{c}}-E_{\mathrm{v}}\right)=0
$$

or in equiponderant form

$$
\nabla_{\boldsymbol{k}}\left[E_{\mathrm{c}}(\boldsymbol{k})-E_{\mathrm{v}}(\boldsymbol{k})\right]=0 .
$$

Expression (8) is fulfilled when

$$
\nabla_{\boldsymbol{k}}\left[E_{\mathrm{c}}(\boldsymbol{k})\right]=\nabla_{\boldsymbol{k}}\left[E_{\mathrm{v}}(\boldsymbol{k})\right]=0
$$

or 


$$
\nabla_{\boldsymbol{k}}\left[E_{\mathrm{c}}(\boldsymbol{k})\right]=\nabla_{\boldsymbol{k}}\left[E_{\mathrm{v}}(\boldsymbol{k})\right] \neq 0 .
$$

The condition (9) is fulfilled in the critical points of BZ in which simultaneously in valence and conduction band the derivative is always equal to zero. This is fulfilled in the local maxima and minima of BZ, respectively. For instance for face centred cubic (fcc) structure the critical points are the high symmetry points $\Gamma$, $X, L$ and so on. The condition (10) is fulfilled around the inflexion points of the valence and conduction bands inside the BZ in which both bands are parallel (for instance in fcc structure in high symmetry directions $\Lambda, \Sigma$ and so on).
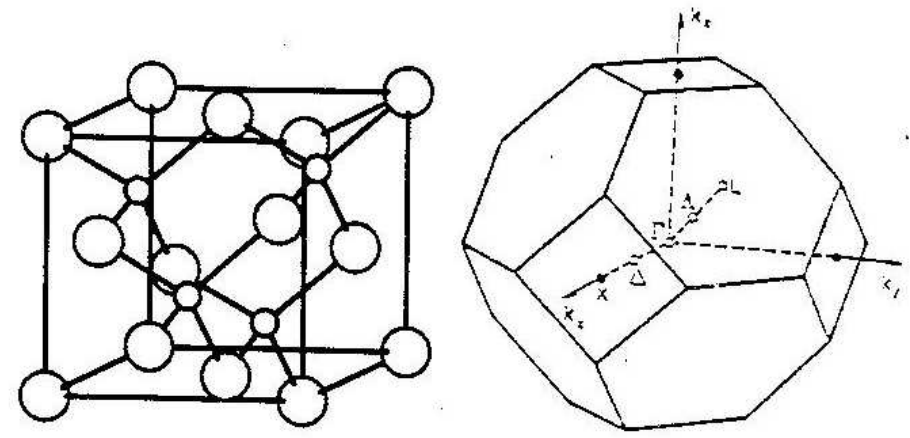

Fig. 1. Zinc blende fcc crystalline structure and the respective first Brillouin zone.

Figure 1 presents fcc crystalline structure in which crystallise a large number of II-VI group semiconducting binary and ternary compounds and corresponding to this structure the first BZ. Latin and Greek letters define the positions of the critical points and distinguished directions of high symmetry in BZ. The conditions (8) and (9) define the position inside the BZ in which the electronic interband transitions probability is higher than in other points of the BZ. An analysis of the singularities in the BZ make easier the identification of the fine structure observed in experimental reflectivity spectrum. A fine structure of the reflectivity spectrum correlated with electronic interband transitions extends from the energy of the forbidden energy gap $E_{\mathrm{g}}$ to about $10 \mathrm{eV}$. In selected cases of wide gap semiconductors and some ionic compounds this limit can be shifted to higher energies.

Figure 2 shows the reflectivity coefficient spectrum for ZnSe [14] being a typical II-VI group semiconducting compound with the forbidden energy gap equal to about $2.7 \mathrm{eV}$. In Fig. 2 there are observed three distinct maxima and some number of smaller maxima and thresholds in the energy range from $2 \mathrm{eV}$ to about $10 \mathrm{eV}$. This fine structure can be identified with the interband transitions at the high symmetry points $\Gamma, L$, and $X$ of BZ and in the high symmetry directions $\Lambda, \Delta, \Sigma$ of BZ $[4,6,14]$. In this figure the experimental reflectivity coefficient spectrum with theoretical linear muffin-tin orbitals (LMTO) calculations [14] has also been compared. 


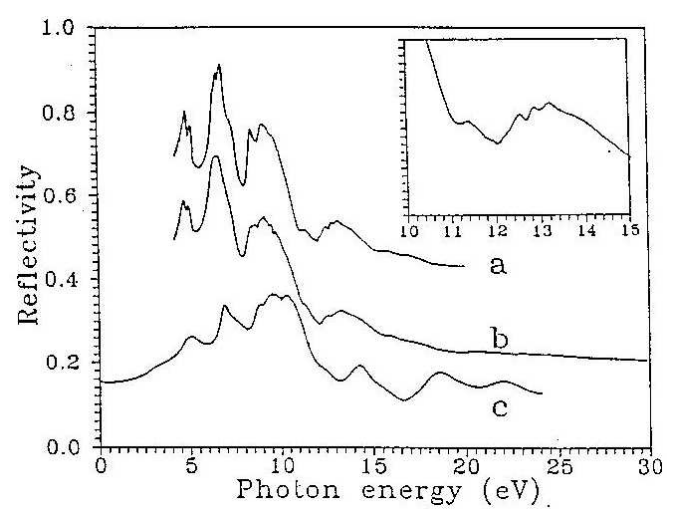

Fig. 2. Reflectivity spectra of ZnSe measured at LNT (curve $a$ ) and RT (curve $b$ ), compared with the theoretical spectrum (curve $c$ ). Curves $a$ and $b$ have been displaced upwards by 0.4 and 0.2 , respectively. The inset shows the region $d_{1}$ of the core transitions measured at LNT on the expanded scale.

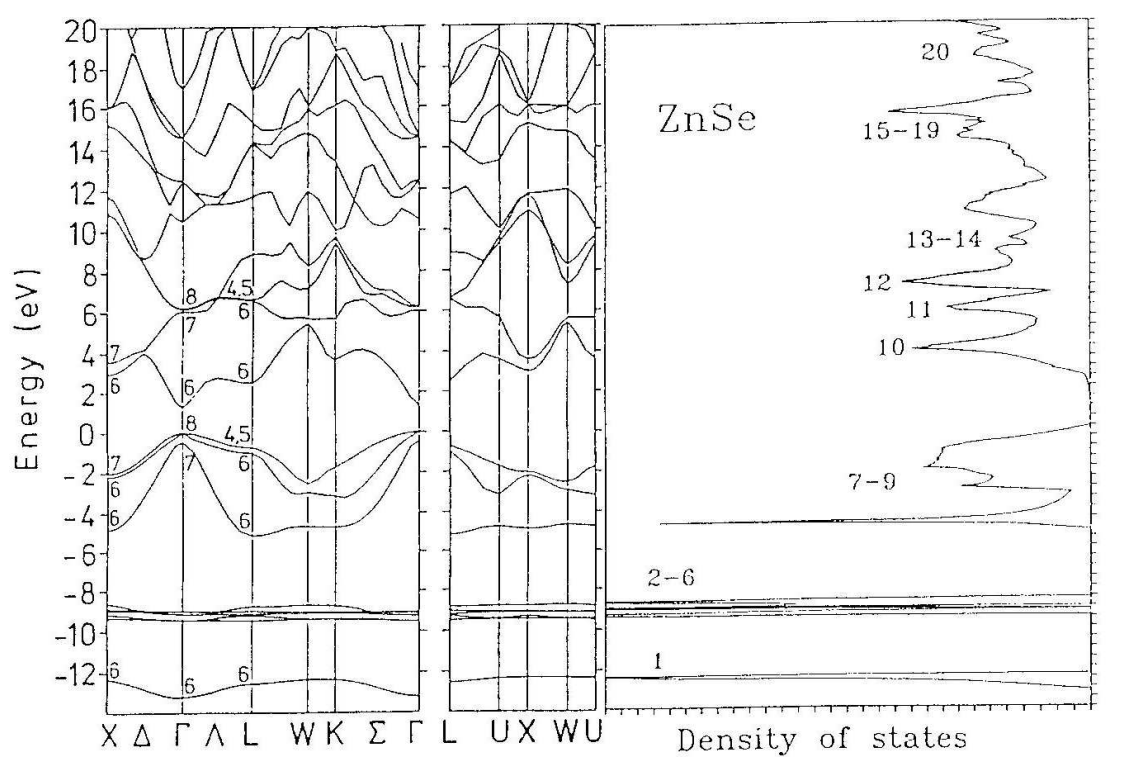

Fig. 3. Band structure of $\mathrm{ZnSe}$. The energy zero is taken to be the top of valence band. The numbers on the left hand figure denote the indexes of the double group notification for selected critical points. On the right-hand side the total density of states was shown. The numbers near the main peaks denote the serial numbers of bands.

Figure 3 shows the band structure and density of states (DOS) energy distribution for ZnSe valence and conduction bands calculated by LMTO method. To demonstrate wave function hybridisation in the electronic structure of $\mathrm{ZnSe}$ Fig. 4 illustrates a schematic layout of the hybridisation energy ranges for $\mathrm{Zn}$ and 


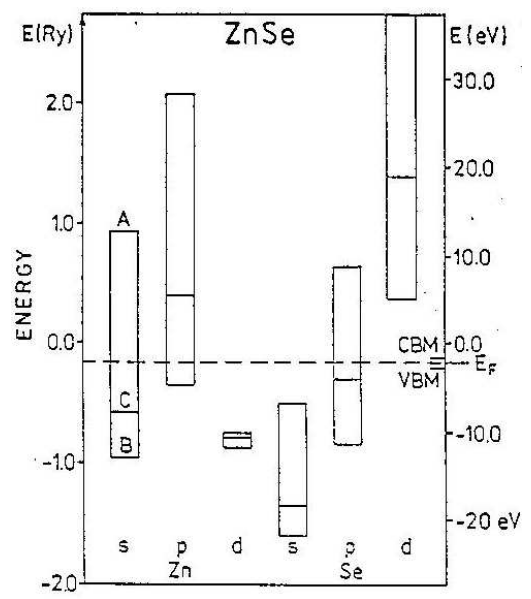

Fig. 4. Schematic layout of the electronic structure of ZnSe. The bars depict the extent of the $n, l$ bands as defined by the parameters $A$ (top of the band), $C$ (band centre) and $B$ (bottom of the band). The valence band maximum (VBM) and the conduction band minimum $(\mathrm{CBM})$ are also marked.

Se $s, p$ and $d$-like functions, respectively. The interband transition probability defined by the JDOS is a convolution of the respective valence band DOS with allowed for transition the conduction band DOS. Therefore, the absorption and reflectivity coefficients in the interband transition energy range deliver only the information on the convoluted valence and conduction band DOS which cannot be separated. This situation is not convenient, nevertheless some important information on the characteristic features of the unoccupied conduction band can be extracted. A direct obtaining of the unoccupied conduction band DOS is possible from the reflectivity spectrum analysis for higher energies (above $10 \mathrm{eV}$ ).

\subsection{Core level electronic transitions to the conduction band}

For the energies above $10 \mathrm{eV}$ the reflectivity spectrum presented in Fig. 2 shows two groups of maxima and thresholds signed by Cardona and Greenaway [15] as $d_{1}$ and $d_{2}$ transitions, respectively. These features are generally connected with transitions from occupied core states $n d^{10}$ and $n s^{2}$ to the projected density of states of unoccupied states of the conduction band. In the case of ZnSe [14] a contribution of the electronic transitions from the initial occupied core states are Zn $3 d^{10}$ ( $d$-like core state) $)^{\dagger}$ and Se $4 s^{2}$ (s-like core state) to the unoccupied DOS is presented in Fig. 2. As shown in Fig. 3, five split core states Zn $3 d^{10}$ (states numbers 2-6) and Se $4 s^{2}$ (state number 1) are rather flat and weakly $\boldsymbol{k}$ dependent. It means that when the initial state is flat and does not depend on the $\boldsymbol{k}$ vector i.e. grad $E_{\mathrm{v}} \equiv 0$, then the join density of states function described by formula (7) depends only on the density of states of the conduction band $E_{\mathrm{c}}$. Thus the

\footnotetext{
${ }^{\dagger} \mathrm{Zn} 3 d^{10}$ ia a part of the $\mathrm{M}$ core level configuration $-3 s^{2} p^{6} 3 d^{10}$.
} 
absorption coefficient $\mu(E)$ is described by a simpler formula in the form

$$
\mu(E)=\left|M_{\mathrm{fi}}\right|^{2} \rho(E),
$$

where $\left|M_{\mathrm{fi}}\right|^{2}$ was defined earlier as the matrix element of initial and final state interaction, and $\rho(E)$ is the density of states of the final state (conduction band).

Formula (11), known as Golden Formula can be applied with sufficient approximation for the transitions from the highest core levels to the conduction band. One should remember that using the Kramers-Kronig relations the reflectivity coefficient can be always expressed by the absorption coefficient. As shown in Fig. 3, the energy of $\mathrm{Zn} 3 d^{10}$ and Se $4 s^{2}$ core states are in practice $\boldsymbol{k}$ independent and thus the density of state for these core states could be approximated with sufficient precision by the Lorentzian shape slightly broadened by the Gaussian broadening. The respective initial core states are narrow, thus the shape of the reflectivity spectrum in $d_{1}$ group of transitions ( $d$-like core state) corresponds directly to the sum of the shape for $p$ - and $f$-like projected density of states in the high symmetry critical points of the conduction band. The shape of $d_{2}$ group of transitions, which are mainly the transitions from $s$-like core states to $p$-like states of the conduction band, reproduce the shape of $p$-like projected density of states summarised in high symmetry critical points of the conduction band.

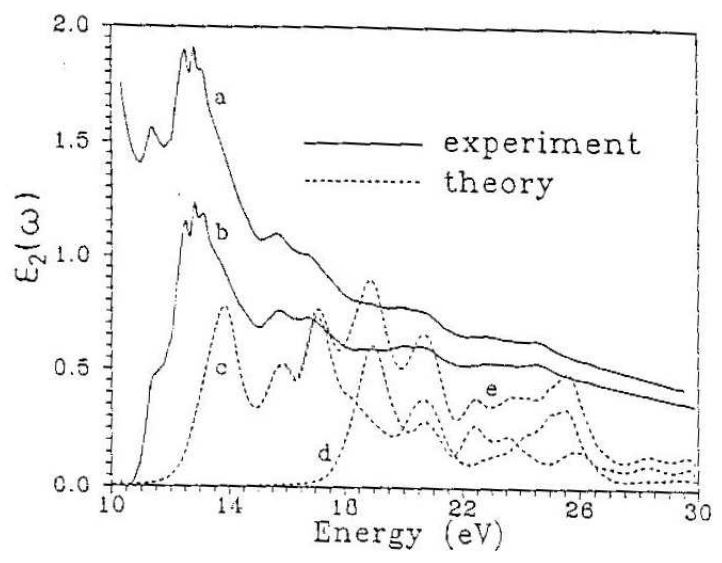

Fig. 5. Imaginary part of the dielectric function for ZnSe between 10 and $30 \mathrm{eV}$ : curve $a$ - LNT experimental spectrum; curve $b$ - spectrum subtracting a smooth exponential background extrapolated from transitions from the valence to conduction band; curve $c$ - theoretical contribution from the $\mathrm{Zn} 3 d$ transitions; curve $d$ - theoretical contribution from the Se $4 s$ transitions; curve $e$ - theoretical contribution from the superposition of Zn $3 d$ and Se $4 s$ transitions (after [11]).

Figure 5 presents the comparison of the liquid nitrogen temperature (LNT) experimental spectrum with the theoretically calculated contributions from the $\mathrm{Zn} 3 d$ and Se $4 s$ transitions, respectively. An agreement of the room temperature (RT) and LNT experimental spectrum with LMTO theoretical calculations shown 


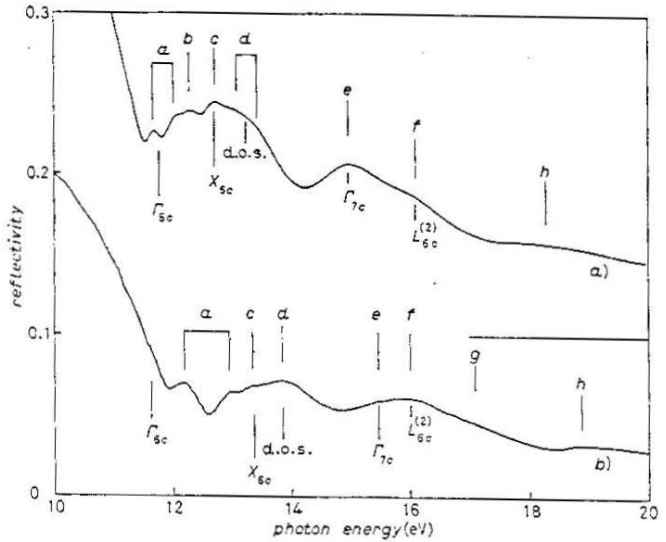

Fig. 6. Reflectivity spectra of curve $a-\mathrm{ZnTe}$ (shifted upwards by 0.1 ) and curve $b$ - CdTe measured at room temperature in the region of the $\mathrm{Cd} 3 d$ excitations. The assignments of the observed structures were identified and collected in table I of Ref. [16].

in Fig. 2 and Fig. 5 is much better for electronic transitions of group $d_{1}$ than for $d_{2}$. The form of the spectrum of the reflectivity coefficient in 10-30 eV energy range for various semiconductors significantly differs. Figure 6 illustrates these differences for $\mathrm{ZnTe}$ and $\mathrm{CdTe}$ semiconductor compounds. A precise identification of the fine structure maxima and thresholds for ZnTe and CdTe indicated in Fig. 6 can be found in Ref. [16]. The sensibility of the reflectivity spectra analysis in the application to the unoccupied conduction band can be presented on the basis of ZnMeSe reflectivity spectrum in which a part of Zn atoms has been replaced by one of the transition metals (Me). As it is well known, the transition metals have only a partially fulfilled $3 d$ electronic subshell. The simplest picture of the replacement of $\mathrm{Zn}$ by a transition metal can be obtained for Mn.

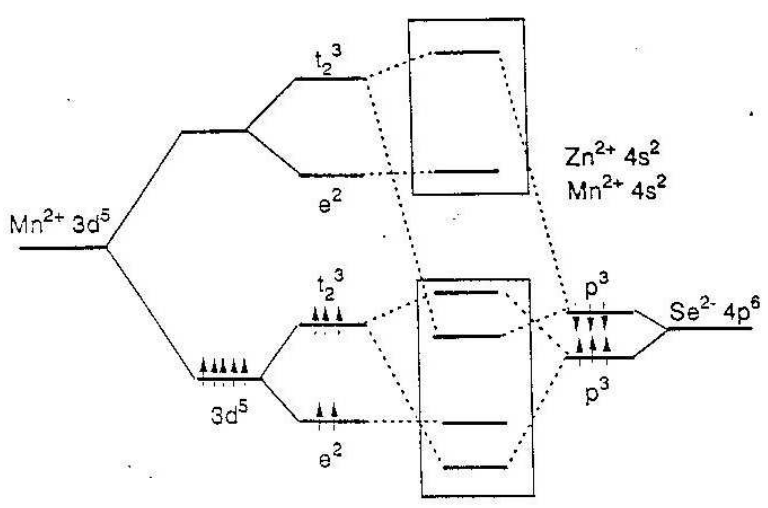

Fig. 7. A schematic energy diagram of the $s, p-d$ coupling for ZnMnSe. 
As shown in Fig. 7, Mn $3 d$ subshell is fulfilled in half $\left(3 d^{5}\right)$ [17]. It is very convenient that a ternary compound ZnMnSe crystallises like ZnSe, in zinc blende (ZB) crystalline structure and thus both ZnMnSe and ZnSe are described by the same shape of the first BZ. Zn $3 d^{10}$ states are situated in pure ZnSe and ZnMnTe behind of the valence band about $9 \mathrm{eV}$ below the top of the valence band maximum (see Fig. 3). In the ternary ZnMnSe the occupied electronic states of the Mn $3 d^{5}$ electrons are situated inside the valence band about $3.5 \mathrm{eV}$ below the top of the valence band at the $\Gamma$ point and the unoccupied Mn $3 d^{5}$ electron states are immersed in ZnSe conductivity band unoccupied states about $2 \mathrm{eV}$ above the main conductivity band minimum at the $\Gamma$ point [18]. Mn $3 d^{5}$ occupied electronic states hybridise with valence electronic states of Zn and Se and strongly modify the pure $\mathrm{ZnSe}$ valence band DOS close to the top of the valence band. Similarly, Mn $3 d^{5}$ unoccupied electronic states modify unoccupied conduction band states DOS of ZnMnSe in the whole conduction band above the forbidden gap energy. An inclusion of the $\mathrm{Mn}$ and other transition metals to all semiconducting materials produces a comfortable condition for the experimental testing, both valence and conduction bands. This procedure allows to observe mainly the distinct modifications of the conduction band DOS for ternary ZnMnSe with the use of the reflectivity spectrum in $10 \mathrm{eV}$ to $30 \mathrm{eV}$ energy range and XANES analysis (see Fig. 8). In the reflectivity spectrum of $\mathrm{ZnMnSe}$, as is presented in Fig. 8 with an increase in $\mathrm{Mn} 3 d^{5}$ states there increases smearing of the fine structure observed for pure $\mathrm{ZnSe}$. A precise identification of the maxima and thresholds has been suggested in Ref. [19]. The smearing effect is observed also for other ternary II-VI compounds with other transition metals dilution [19].

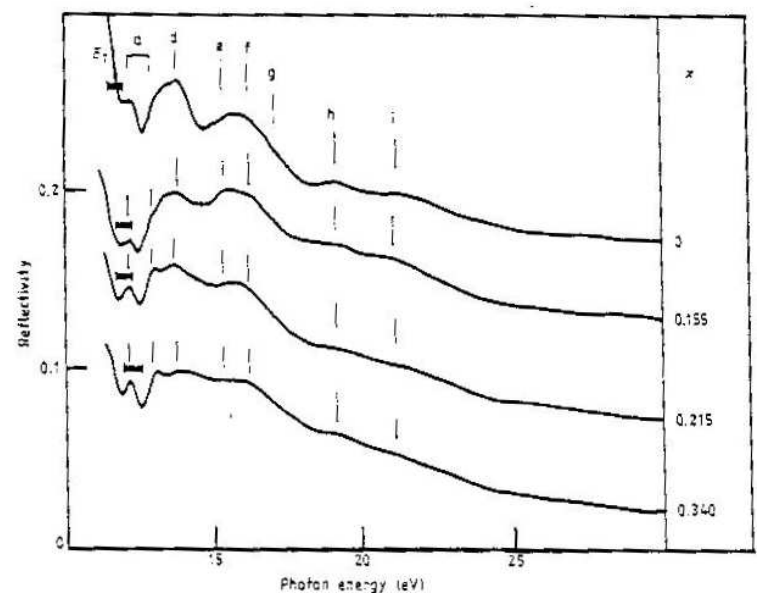

Fig. 8. Reflectivity spectra of CdTe and $\mathrm{Cd}_{1-x} \mathrm{Mn}_{x}$ Te in the $10-30 \mathrm{eV}$ energy range. The marks of the observed fine structure were identified in Ref. [16]. 


\section{Analysis of the X-ray absorption near edge structure}

In the experimental X-ray absorption spectrum of solid state in various energy ranges there are observed absorption edges which are a result of the electronic transitions from deep $K, L$ or $M$ and so on core levels to the unoccupied states of the solid state conduction band. The result of these transitions are steep absorption edges with a fine structure known as XANES. These transitions carry information on the basic features of the conduction band unoccupied states and can very precisely characterise the DOS of the conduction band empty states in the whole first BZ. The deep $K$ and $L$ core levels are described by $s, p$-type quantum functions, respectively, thus in the real material the transitions from these levels to the hybridised DOS of the conduction band are allowed if adequate selection rules are fulfilled. This part of the total DOS to which respective electronic transitions are allowed are named the projected density of states (PDOS) of the conduction band. In the dipole approximation, the electron transitions from the core states to the respective selected PDOS are allowed when selection rules $\Delta l= \pm 1$ and $\Delta j=0, \pm 1$ are fulfilled.

The electronic transitions from the $K$ core level occupied by $1 s^{2}$ electrons are characterised by the orbital momentum quantum number $l=0$, spin number $s= \pm 1 / 2$ and total momentum $j= \pm 1 / 2$. From this level, which is an $s$-type level, there are allowed transitions only to the $p$-like PDOS of the conduction band. For the $L$ core level occupied by $2 s^{2} 2 p^{6}$ electrons, which is described by the orbital quantum number $l=0,1$, and $s= \pm 1 / 2$ and $j= \pm 1 / 2, \pm 3 / 2$ the electronic transitions create three following edges:

$L_{1}-l=0, j= \pm 1 / 2$ i.e. $s$-type sublevel to $p$-like PDOS,

$L_{2}-l=1, j=+1 / 2$ i.e. $p$-type sublevel to $s(j=-1 / 2)$ - and $d(j=$ $+1 / 2)$-like PDOS,

$L_{3}-l=1, j=+3 / 2$ i.e. $p$-type sublevel to $s(j=+1 / 2)$ - and $d(j=$ $+3 / 2)$-like PDOS,

Partial studies of transitions from $M$ subshell in $\mathrm{ZnTe}$ with the use of the fundamental reflectivity analysis (only the transitions from $3 d^{10}$ subshell to the conduction band) have been described in the previous section. The discussion on the transitions from $\mathrm{N}$ and $\mathrm{O}$ and so on core levels to the conduction band is realised in the materials containing heavy elements, thus in this paper has been omitted. The absorption coefficient of transitions from the $\boldsymbol{k}$ momentum vector independent $K$ and $L_{1}, L_{2}$ and $L_{3}$ core levels, to the unoccupied states of conduction band are described by the golden formula (9).

The XANES energy range extends from about $10 \mathrm{eV}$ below the steep of $\mathrm{X}$-ray absorption edge (pre-edge structure) to about $20 \mathrm{eV}$ above this edge. Each chemical element is characterised by the specific absorption edge energy position which allows an identification of the element and an analysis of the conduction band DOS distribution around each selected ion in the solid state. In the study of the absorption edge in the XANES energy range two aspects are analysed: 
(1) the shape and position of the edge, and

(2) the fine structure on and around the absorption edge.

\subsection{Shape and position of absorption edge}

Richtmyer et al. [20] have shown that an ideal X-ray absorption edge can be described by arctangent function which is the result of the transition from Lorentzian shape core state to the constant (non-zero) in the whole conduction band DOS. This theoretical consideration was confirmed experimentally on the $K$ absorption edge of $\mathrm{Au}$ which has an almost flat conductivity band DOS distribution [20]. For the ideal absorption edge the Fermi energy level or chemical potential are localised at the inflection point of arctangent function. In this point the first derivative has a maximum which defines the position of the Fermi energy level on the ideal experimental edge. For the real X-ray absorption edges which describe the convolution of the Lorentzian shape core state with a complicated DOS distribution of the conduction band this definition on the Fermi energy level position is only approximate. If the description of the Fermi level position makes some difficulties, then for a comparison of various edges, sometimes it is better to use the first distinct maximum (a white line) in the XANES spectrum. For the various compounds of the same chemical element, the energy position of the $\mathrm{X}$-ray absorption edges can differ even by several $\mathrm{eV}$ in comparison to the energy position for the pure element. The problem is rather complex because of several components which may have an influence on such a shift. The most essential component is the oxidation state of the analysed element in the chemical compound. Generally, the oxidation state can be defined by the thermodynamic functions of the chemical compound and by the symmetry of the solid state crystalline structure. The conditions of the thermodynamic equilibrium define a characteristic chemical potential for a solid state i.e. Fermi energy level position at $0 \mathrm{~K}$. A shift of the absorption edge energy positions connected with the changes of oxidation state is well known. For the application purposes Hormes et al. [21] reported an analysis of the energy shifts of $\mathrm{Cr} K$ edges on the oxidation state in various chromium compounds. They found a linear dependence of the $\mathrm{Cr} K$ absorption edge energy position on the 3, 4, 5 and 6 valency (oxidation) state which allows a correct diagnosis of $\mathrm{Cr}$ oxidation state in not well known compounds. Besides, an application of the oxidation state definition in the biological materials makes a physical diagnostic of these materials easier. Kwiatek et al. [22, 23] studied the energy shifts of Fe $K$ edges in non-cancerous and cancerous prostate tissues and found that $\mathrm{Fe}$ in these materials appears in III and II oxidation states, respectively. This suggestion has been confirmed by an analysis of the Fe $K$ edge positions for the standard materials with Fe being on different oxidation state (metallic $\mathrm{Fe}$, fayalite, magnetite and hematite) $[22,23]$. Sulphur is commonly the component of the biological proteins, therefore also $\mathrm{S} K$ absorption edge can deliver valuable information on these materials. 


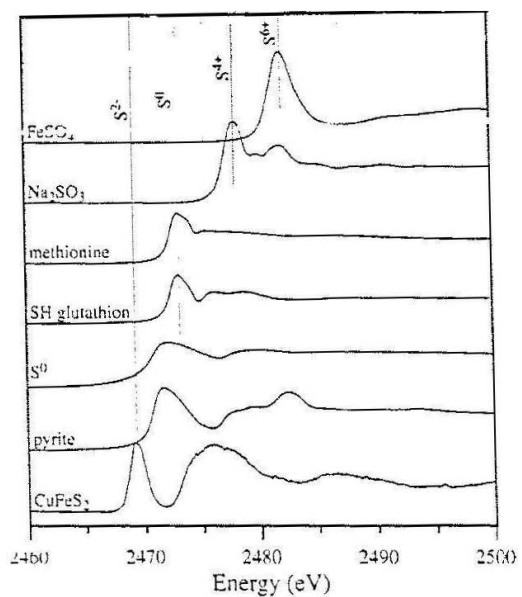

Fig. 9. Sulphur $K$ absorption edges positions in the compounds with various oxidation states.

Figure 9 presents a shift of the $\mathrm{S} K$ absorption edge in the standard compounds in which sulphur has various oxidation states. This dependence has been applied to the diagnosis of the bacterial filaments and the fossils of the microorganisms [24]. Similar standard sulphur compound series were used for the analysis of the non-cancerous and cancerous tissues [25] and cultured human prostate cancer cells [26]. In the compounds in which the oxidation state does not change, one can observe smaller modifications of the shape and absorption edge energy position. These types of changes are discussed below.

\subsection{Fine structure of the X-ray absorption edge}

As mentioned above, the fine structure of the X-ray absorption edges can appear before the absorption edge as a pre-edge structure, on the steep part of the absorption edge and also above the edge in form of maxima and thresholds. To illustrate these features, selected and characteristic examples of the $K$ and $L$ X-ray absorption edges for some II-VI binary and ternary with transition metals semiconducting compounds have been presented.

A fine structure of the Te $L_{1}$ and $L_{3}$ absorption edges and their first derivatives for ZnTe, CdTe and HgTe semiconducting compounds are shown in Fig. 10 [9]. ZnTe, CdTe and HgTe crystallise in the cubic ZB structure and therefore these crystals are represented by identical first Brillouin zone. It is clear that the total DOS and respective PDOS energy distributions for ZnTe, CdTe and HgTe are different. These differences induced by various $\mathrm{Zn}, \mathrm{Cd}$ and $\mathrm{Hg}$ atomic electronic structure are a result of the respective modifications in the spectrum of Te $L_{3}$ and $L_{1}$ edges for all of these materials. In the chemical compounds of the II-VI group the distribution of the bond charge around the elements is commonly asymmetric [14]. Therefore, it is not strange that the DOS and each PDOS energy distributions around each atom or ion in the same compound depend on the charge 

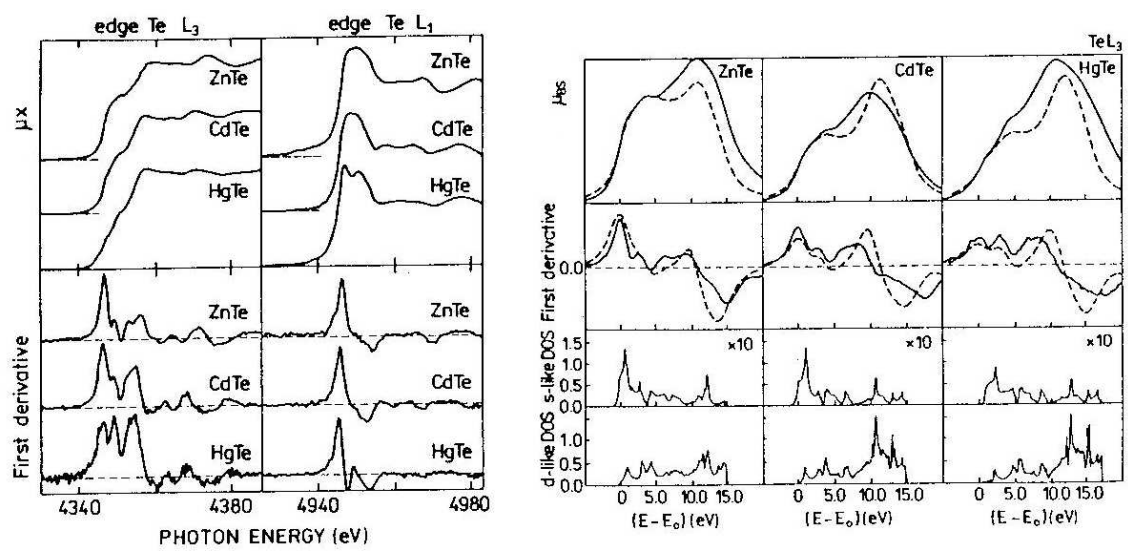

Fig. 10. Left figure - Te $L_{3}$ and $L_{1}$ X-ray absorption edges and their first derivatives for $\mathrm{ZnTe}$, CdTe and HgTe. Right figure — reduced experimental spectrum (solid line) with theoretical convolution (dashed line) and their first derivatives for $L_{3}$ edges. Below, the calculated projected $s$ - and $d$-like conductivity band DOS.
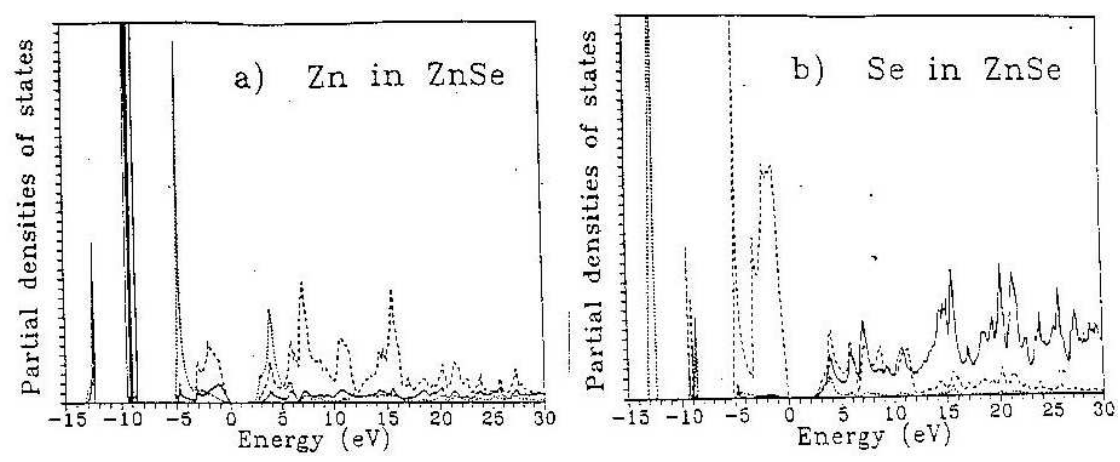

Fig. 11. (a) Projected DOS at a Zn site, (b) projected DOS at a Se site: $\cdots s$-like states, - - p-like states, - $d$-like states.

distribution around the compound constituents. This situation is shown in Fig. 11 for $\mathrm{ZnSe}$ - another member of the II-VI group. Respective PDOS around Zn and Se site are significantly different. XANES as a selective method of analysis is sensitive to these differences. Looking at the first derivative maximum of Te $L_{3}$ and $L_{1}$ edges (see Fig. 10) for ZnTe, CdTe and HgTe it is easy to see that the energy positions of the inflation points for all edges are in practice not shifted. To obtain more complete information on the conduction band unoccupied states it is necessary to compare experimental XANES data with the theoretical band structure calculations. The energy range of the theoretical calculations with sufficient high precision is strictly limited. Therefore, a valuable comparison of the band structure calculation results with the experimental data known in a wide energy range is easier with applying the special reduction procedure described in 
Refs. [9] and [7]. The reduction procedure consists on the cut of the experimental data in the energy range in which the theoretical calculations are sufficiently credible. Figure 10 presents a comparison of the LMTO theoretical calculations of $L_{1}$ and $L_{3}$ edges for $\mathrm{ZnTe}, \mathrm{CdTe}$ and $\mathrm{HgTe}$ with the respective, reduced experimental data. Some small differences are caused by a specific PDOS energy distribution around $\mathrm{Zn}$ and $\mathrm{Cd}$ ions. As mentioned earlier (Sect. 2.2), the analysis of the ternary compounds with transition metals is a source of interesting information about properties because the $s-p-d$ hybridised DOS from partially fulfilled $3 d$ subshells of the transition metals are localised closely to the Fermi level. Thus they are a good indicator of the sensitivity of the XANES analysis. Similarly to the previous chapter the XANES analysis has been focused mainly on ZnSe and its ternary compounds. Unoccupied states of the $3 d$ subshell of $\mathrm{Mn}\left(3 d^{5}\right)$ and Fe $\left(3 d^{6}\right)$ are situated closely to the bottom of the conduction band of the pure ZnSe and hybridise with the respective $s, p$ and $d$ states of Zn and Se (see Fig. 7). A result of the spd hybridisation is very well observed in $\mathrm{Zn}$ and Se $K$ edges spectrum for ZnMnSe and ZnFeSe and other transition metals. The contribution of the $3 d$ unoccupied states hybridisation of $\mathrm{Mn}$ and Fe in $p$-like PDOS of ZnMnSe and $\mathrm{ZnFeSe}$ has been observed.

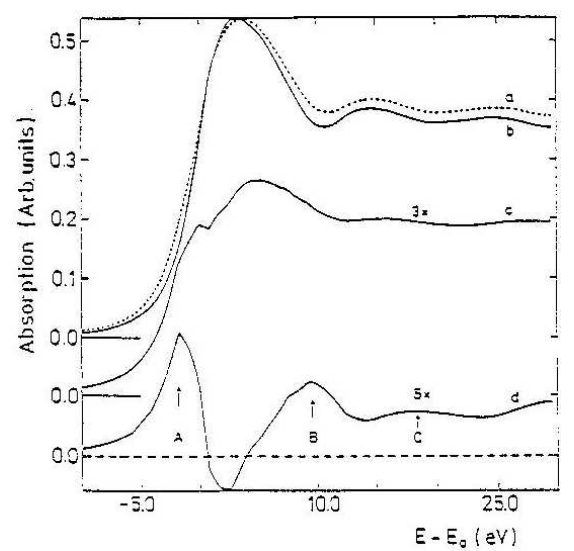

Fig. 12. An analysis of the experimental X-ray absorption Se $K$ edges for ZnFeTe. Curve $a$ (dotted line) - absorption coefficient for ZnFeSe in reduced energy scale, curve $b$ (solid line) - absorption coefficient for ZnSe, curve $c$ - hypothetical absorption edge for ZB crystalline structure FeSe, curve $d-$ a contribution of the hybridised $\mathrm{Fe}$ $3 d$ and $4 s$ electronic states in $\mathrm{ZnFeSe}$.

Figure 12 shows Se absorption edges for ZnSe and ternary ZnFeSe. The edges for $\mathrm{ZnSe}$ and for $\mathrm{ZnFeSe}$ are very similar but small differences are very closely localised to the inflection point. Extracted differences in experimental Se $K$ edge spectrum of ZnFeSe show that the contribution of the $s p d$ hybridisation induced by transition metal, modifies $p$-like PDOS of Se. Figure 12 illustrates the localisation 


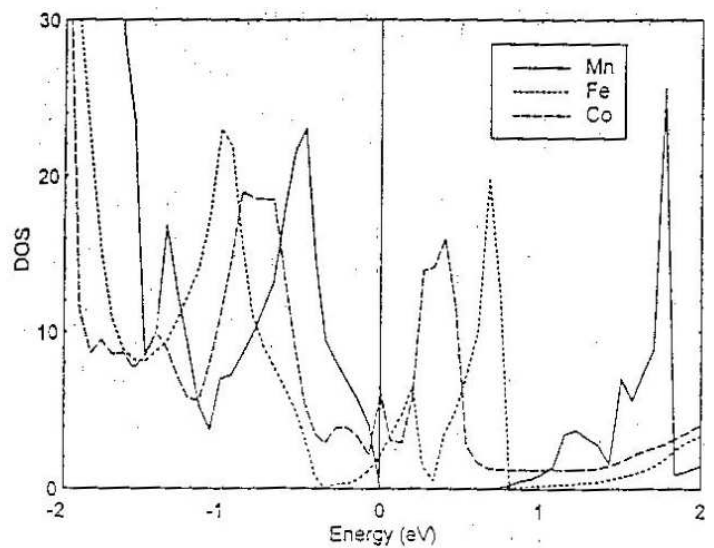

Fig. 13. Theoretical p-PDOS around the Fermi energy level for ZnMnSe, ZnFeSe and $\mathrm{ZnCoSe}$.
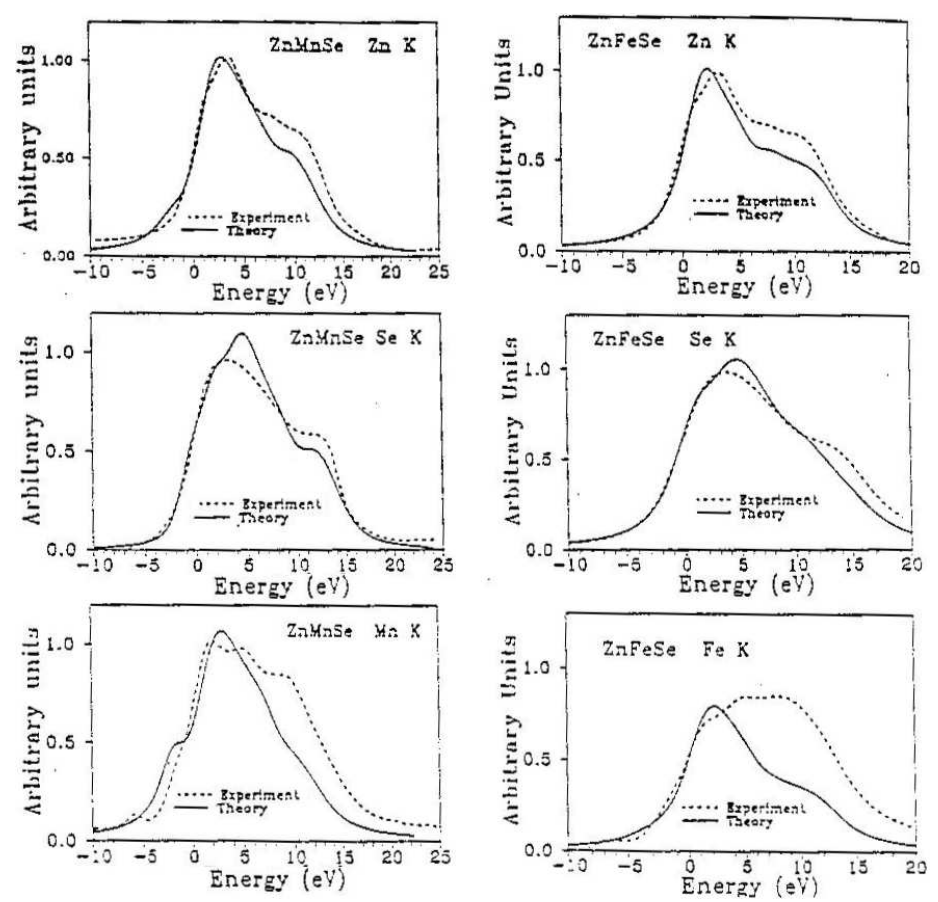

Fig. 14. A comparison of reduced experimental spectrum (dashed line) with theoretical convolution (solid line) for $\mathrm{Zn}$, Se, Mn and Fe $K$ edges in ZnMnSe and ZnFeSe.

of the main contribution of the Fe spd hybridisation in ZnSe closely to the Fermi level. The contribution of the $s p d$ hybridisation is different for various transition metals as has been shown in Fig. 13. This figure presents LMTO theoretical calculations of $p$-like DOS around Se ion for ZnMnSe, ZnFeSe and ZnCoSe near the 
Fermi level. The theoretical calculations for ZnFeTe are in a very good agreement with the experimental data analysis shown in Fig. 12d spectrum. A comparison of reduced experimental results for ZnMnSe and ZnFeSe with LMTO theoretical calculations for $K \mathrm{Zn}$, Se, Mn and Fe absorption edges are shown in Fig. 14.

It is well known that the crystalline structure of the material have significant influence on the occupied and unoccupied states DOS energy distribution. It means that DOS energy distribution depends on the shape of the first Brillouin zone. A comparison of the XANES results for fcc cubic structure $\mathrm{ZnS}$ and wurzite (hexagonal) structure CdS shows a significant shift of the white line in the sulphur $K$ edge XANES spectrum [26]. This is also easily seen by looking at the theoretical band structure calculations for CdSe in hexagonal and zinc blende structures. Both modifications of CdSe possess various first BZs and therefore should have different DOS energy distributions. Figure 15 presents the evident differences of the DOS energy distribution for both kinds of CdSe structures [27] and thus one could expect that the fine structure of the XANES spectrum should also be different. Unfortunately, the experimental XANES data are missing that could confirm this evident suggestion.

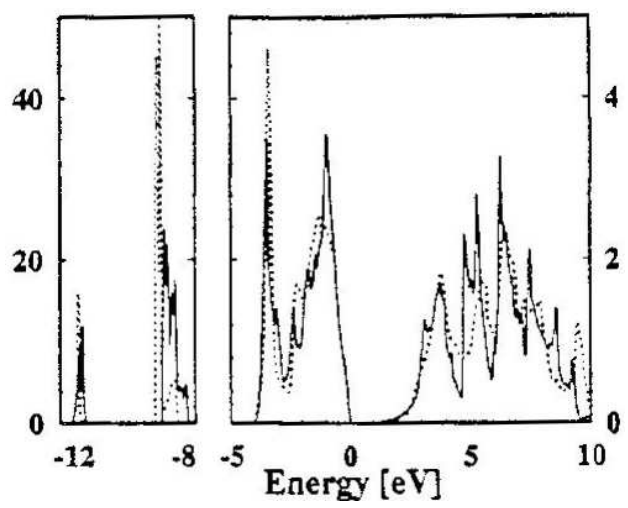

Fig. 15. Variation of the total DOS for CdSe calculated Korringa-Kohn-Rostoker (KKR) method: solid line — wurzite (hexagonal) structure and dashed line — zinc blende structure.

XANES studies can deliver information on the spin distribution in selected materials even without application of the magnetic dichroism analysis. An interesting example of the spin up and spin down distribution analysis can be reached for $\mathrm{CuCr}_{2-x} \mathrm{Sb}_{x} \mathrm{~S}_{4}(x=0.3,0.4$ and 0.5) [28]. These compounds for $x=0.3$ and 0.4 are ferromagnetic $p$-type metals and for $x=0.5$ are antiferromagnetic semiconductors. This evolution of the magnetic properties has been studied by XANES for the S $K$ absorption edges [28].

Figure 16 shows the XANES of the $\mathrm{S} K$ absorption edges for $\mathrm{CuCr}_{2-x} \mathrm{Sb}_{x} \mathrm{~S}_{4}$ $(x=0.3,0.4$ and 0.5$)$. The intensity of the threshold $A$ decreases with the increase 


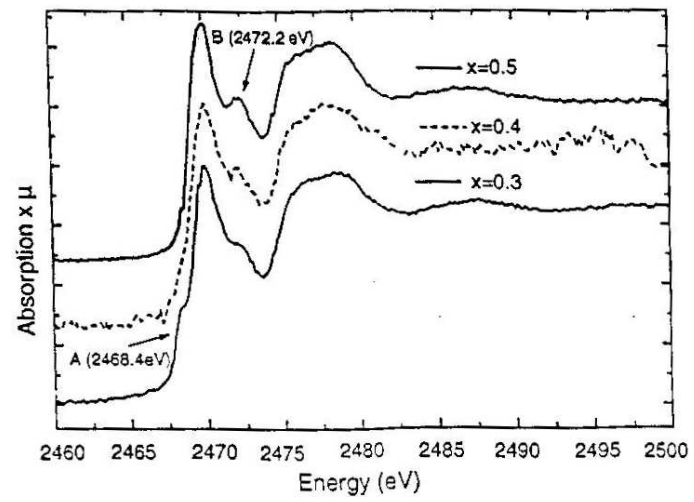

Fig. 16. Experimental spectra of X-ray absorption edges of sulphur for $\mathrm{CuCr}_{2-x} \mathrm{Sb}_{x} \mathrm{~S}_{4}$ $(x=0.3,0.4$ and 0.5$)$.

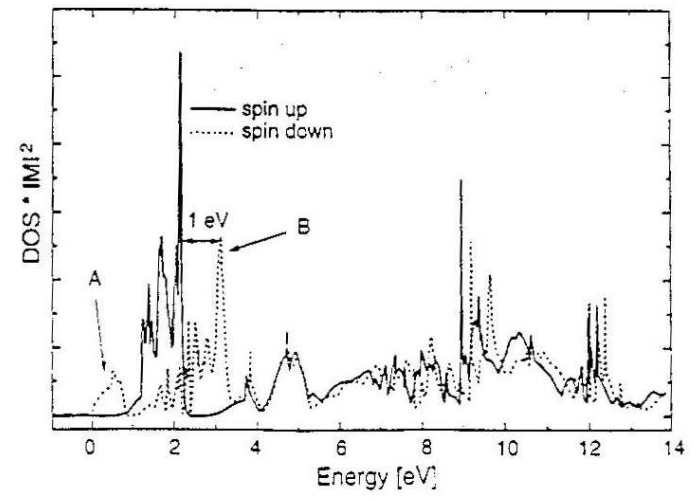

Fig. 17. Theoretical projected spin-dependent $p$-like DOS around sulphur for $\mathrm{CuCr}_{1.5} \mathrm{Sb}_{0.5} \mathrm{~S}_{4}$; solid line — spin up and dashed line — spin down.

in $\mathrm{Sb}$ content whereas the intensity of maximum $B$ increases with the increase in Sb content. The application of the spin dependent linear augmented plane waves (LAPW) method to the analysis of the sulphur $p$-like PDOS suggests (see Fig. 17) that the evolution of the XANES $S K$ edge spectrum with composition of Sb by the polarisation of electron spins on the sulphur is affected.

In the XANES spectrum there is often observed a pre-edge structure which contains one or, as in the case of $\mathrm{Ti}$, even several maxima. The nature of this structure is sometimes correlated with less probable dipole-magnetic or electric-quadrupole transitions from the core state to the respective PDOS of the unoccupied conduction band. For free light atoms and ions the dipole-magnetic or electric-quadrupole transitions are strongly forbidden. In the solid state this is partially broken but the probabilities of this type transitions are also small. Muller and Wilkins [29] for the solid states with the elements lighter than $\mathrm{Cu}$ estimated that the contribution of the electric-quadrupole in the electric-dipole in $K$ edge 
transition is smaller than about $6 \%$. For $L$ edges this ratio is even several times smaller. The XANES theoretical analysis of the selenides with Fe, Co and Ni [30], which are lighter than $\mathrm{Cu}$, shows that partially empty $3 d$ states of transition metals can produce the PDOS energy distribution very closely to the Fermi level. This effect has been also confirmed for other transition metals.

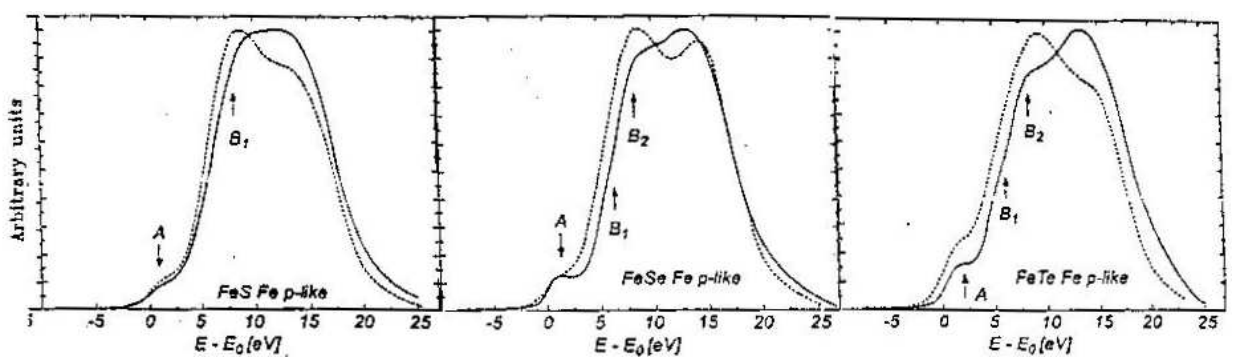

Fig. 18. Reduced XANES spectra and LMTO calculations $p$-like DOS convoluted with Lorentzian and Gaussian functions for FeS, FeSe and FeTe; experiment — solid lines, theoretical calculations - dashed lines.

Figure 18 presents the comparison of the reduced experimental XANES spectra for FeS, FeSe and FeTe with the theoretical LMTO calculations. The band structure calculations reproduce the sufficiently well defined pre-edge structure observed in the experiment. This comparison confirms that the simple pre-edge structure can be, at least for the transition metal halcogenides, explained by the electric-dipole transitions from the transition metal $K$ core states to the unoccupied states of the conduction band.

\section{Conclusions}

The paper is devoted to the unoccupied states analysis for the selected number of the semiconducting materials with the use of the intense synchrotron radiation. The experimental and theoretical studies of the DOS for unoccupied conduction band are a valid source of knowledge on the electronic structure of condensed matter. The fundamental reflectivity of light in a wide energy range and the XANES analysis as the most effective synchrotron radiation experimental methods were discussed. The analysis of the fundamental reflectivity spectrum provides valid information on the transitions from the electronic occupied valence band to the unoccupied states of conduction band (interband transitions) and on the transition from the highest electronic core levels to the DOS of the unoccupied conduction band in the solid state. XANES analysis provides information on the transition from deep electronic core levels to the DOS of unoccupied conduction band. The reasons of the complex structure around the X-ray absorption edges have been considered. In the paper the menaces of the correct interpretation of the experimental results were discussed. XANES experimental results and an analysis of fundamental reflectivity of light have been presented for several binary and 
ternary semiconductor compounds of II-VI group with Mn, Fe and Co. Moreover, some applications of the XANES analysis to some inorganic compounds and some biological systems were mentioned. To avoid the misinterpretation of the experimental results, the data have been compared and discussed with adequate theoretical band structure calculations.

\section{References}

[1] Semiconductors, Ed. N.B. Hanney, Reinhold Publ. Co., New York 1959.

[2] C. Kittel, Introduction to Solid State Physics, Wiley, New York 1997.

[3] N.W. Ashcroft, N.D. Mermin, Solid State Physics, Holt, Rinehart, Winston, New York 1976.

[4] J.C. Phillips, Solid State Phys. 18, 58 (1966).

[5] T.S. Moss, G.J. Barrell, B. Ellis, Semiconductor Opto-Electronics, Butterworth \& Co.Ltd, New York 1973.

[6] F. Bassani, G. Pastori-Paravicini, in: Electronic States and Optical Transitions in Solids, Ed. R.A. Ballinger, Pergamon Press, Oxford 1975.

[7] L.G. Parratt, Rev. Mod. Phys. 31, 616 (1959).

[8] J.E. Muller, J.W. Wilkins, Phys. Rev. B 29, 4331 (1984).

[9] A. Kisiel, G. Dalba, P. Fornasini, M. Podgorny, J. Oleszkiewicz, F. Rocca, E. Burattini, Phys. Rev. B 39, 7895 (1989).

[10] Unoccupied Electronic States, Fundamentals for XANES, EELS, IPS and BIS, Eds. J.C. Fuggle, J.E. Ingelsfield, Springer Verlag, Berlin 1992.

[11] G. Margaritondo, Introduction to Synchrotron Radiation, Oxford University Press, New York 1988.

[12] Synchrotron Radiation. Techniques and Applications, Topics in Current Physics, Vol. 10, Ed. C. Kunz, Springer-Verlag, Berlin 1979.

[13] A. Kisiel, Bull. Polish Synchrotron Radiation Society 6, 145 (2006).

[14] R. Markowski, M. Piacentini, D. Dȩbowska, M. Zimnal-Starnawska, F. Lama, N. Zema, A. Kisiel, J. Phys. Condens. Matter 6, 3207 (1994).

[15] M. Cardona, D.L. Greenaway, Phys. Rev. 131, 98 (1963).

[16] A. Kisiel, M. Zimnal-Starnawska, F. Antonangeli, M. Piacentini, N. Zema, Nuovo Cimento 8D, 436 (1986).

[17] A. Kisiel, M. Piacentini, D. Dȩbowska, N. Zema, F. Lama, M. Zimnal-Starnawska, W. Giriat, A. Hołda, R. Markowski, J. Phys., Condens. Matter 9, 8767 (1997).

[18] J. Oleszkiewicz, M. Podgórny, A. Kisiel, E. Burattini, Phys. Rev. B 60, 4920 (1999).

[19] A. Kisiel, M. Piacentini, F. Antonangeli, J. Oleszkiewicz, A. Rodzik, N. Zema, A. Mycielski, J. Phys. C, Solid State Phys. 20, 5601 (1987).

[20] E.K. Richtmyer, S.W. Barnas, E. Ramberg, Phys. Rev. 46, 843 (1934).

[21] J. Hormes, R. Chauvistre, W. Schmitt, M. Pantelouris, Acta Phys. Pol. A 82, 37 (1992).

[22] W.M. Kwiatek, A.L. Hanson, C. Paluszkiewicz, M. Gałka, M. Gajda, T. Cichocki, J. Alloys Comp. 362, 83 (2004). 
[23] W.M. Kwiatek, A. Banaś, K. Banaś, M. Gajda, M. Gałka, G. Falkenberg, T. Cichocki, J. Alloys Comp. 401, 178 (2005).

[24] J. Foriel, P. Philippot, J. Susini, P. Dumas, A. Somogyi, M. Salome, H. Khodja, B. Menez, Y. Fouquet, D. Moreira, P. Lopez-Garcia, Geochim. Cosmochem. Acta 68, 1561 (2004).

[25] W.M. Kwiatek, A. Banaś, K. Banaś, A. Kisiel, G. Cinque, G. Falkenberg, Acta Phys. Pol. A 109, 383 (2006).

[26] W.M. Kwiatek, M. Podgórczyk, Cz. Paluszkiewicz, A. Balerna, A. Kisiel, Acta Phys. Pol. A 114, 463 (2008).

[27] J. Konior, S. Kaprzyk, Acta Phys. Pol. A 87, 269 (1995).

[28] P. Zajdel, A. Kisiel, J. Warczewski, J. Konior, L.I. Koroleva, J. Krok-Kowalski, P. Gusin, E. Burattini, G. Cinque, A. Grilli, R.V. Demin, J. Alloys Comp. 401, 145 (2005).

[29] J.E. Muller, J.W. Wilkins, Phys. Rev. B 29, 4331 (1984).

[30] A. Kisiel, P. Zajdel, P.M. Lee, E. Burattini, W. Giriat, J. Alloys Comp. 286, 61 (1999) 